\title{
EFEITO DA EXPLORAÇÃO NA RIQUEZA FLORÍSTICA E DIVERSIDADE EM UMA FLORESTA ECOTONAL DA REGIÃO NORTE MATO-GROSSENSE
}

\author{
Chirle Colpini ${ }^{1}$, Versides Sebastião de Moraes e Silva ${ }^{2}$, Thelma Shirlen Soares ${ }^{3}$, \\ José Vespasiano Lisboa Assumpção ${ }^{1}$, Roberto Chiaranda ${ }^{2}$ \\ ${ }^{1}$ Eng. Florestal, Mestrando em Ciências Florestais e Ambientais, UFMT - chcolpini@gmail.com; onf@terra.com.br \\ ${ }^{2}$ Eng. Florestal, Dr., Depto. de Engenharia Florestal, UFMT - versides@ufmt.br; gatotato@uol.com.br \\ ${ }^{3}$ Eng $^{\mathrm{a}}$ Florestal, Dr ${ }^{\mathrm{a}}$., Depto. de Engenharia Florestal, UFVJM - thelsoares@ terra.com.br \\ Recebido para publicação: 01/12/2008 - Aceito para publicação: 13/08/2010
}

\begin{abstract}
Resumo
O objetivo deste estudo foi avaliar as mudanças ocorridas na riqueza e diversidade de espécies em uma floresta ecotonal na região norte mato-grossense. Os dados foram coletados em três ocasiões (2001, antes da exploração, e em 2003 e 2007, após a exploração), em 74 parcelas de 0,25 ha, sendo 69 para estudar a floresta não explorada e 5 para a explorada, com a retirada dos fustes de árvores com diâmetros comercialmente aceitos no mercado. Todos os indivíduos com diâmetro a $1,3 \mathrm{~m}$ de altura do solo (Dap) $\geq 17 \mathrm{~cm}$ foram mensurados e identificados. A diversidade florística foi avaliada por meio do quociente de mistura de Jentsch e pelo índice de Shannon-Wiener e a equabilidade pelo índice de Pielou. A variação da riqueza e diversidade de espécies antes e após a exploração madeireira foi pequena, o que confirma o baixo impacto da exploração na composição e diversidade das espécies da floresta.

Palavras-chave: Diversidade; equabilidade; Amazônia Meridional.
\end{abstract}

\begin{abstract}
Logging effects on floristic richness and species diversity in an ecotonal forest in northern region of Mato Grosso state, Brazil. The aim of this research was to evaluate changes in relation to floristic richness and species diversity in an ecotonal forest in northern region of Mato Grosso State, Brazil. Data had been collected along three different moments (2001 [before logging], in 2003 and 2007 [after logging]) in 74 0.25-ha plots (69 in unlogged forest and 5 in logged forest). Trees with diameter $\geq 17 \mathrm{~cm}$ and $1.3 \mathrm{~m}$ height ( $\mathrm{dbh}$ ) had been measured and identified. Floristic diversity was evaluated by Jentsch Coefficient of Mixture and Shannon-Wiener index as well as its equability by Pielou's Index. There were little variations before and after logging in relation to floristic richness and species diversity, it confirms low impact of exploration on floristic composition and species diversity of the forest.
\end{abstract}

Keywords: Diversity; equability; Southern Amazonia.

\section{INTRODUÇÃO}

A maioria das florestas nativas da Amazônia tem sido explorada de forma não sustentável, sem aplicação dos critérios de sustentabilidade do manejo florestal, o que resulta na perda da cobertura florestal e da diversidade de espécies, antes mesmo que se tenha o conhecimento dessa riqueza natural (SOUZA et al., 2006).

Apenas uma pequena parcela do setor madeireiro na Amazônia adotou o manejo florestal como técnica no processo de intervenção no ecossistema. A grande maioria ignorou ou não reconheceu a importância do manejo na sustentabilidade da produção madeireira e na conservação dos processos estruturais e funcionais das florestas. Dentre os motivos da não adoção do manejo florestal, destaca-se a carência de difusão das informações, conhecimentos científicos e tecnológicos, uma vez que há pouco investimento em pesquisas e elas estão concentradas de forma pontual em institutos de pesquisas e universidades. 
Mesmo considerando os avanços da pesquisa florestal na Amazônia durante as últimas décadas, vários componentes do ecossistema ainda necessitam ser investigados. Como exemplo, pode-se citar a dinâmica florestal, a qual depende de um período mais longo de observação, quase sempre pelo estabelecimento de parcelas permanentes, e exige aporte considerável de pessoal qualificado e recursos (SANQUETTA et al., 2001).

O conhecimento da composição e da riqueza florística, da diversidade de espécies e da estrutura em florestas tropicais é de suma importância para o planejamento e estabelecimento de sistemas de manejo com produção sustentável, bem como para a condução da floresta a uma estrutura balanceada, além de técnicas silviculturais adequadas baseadas na ecologia de cada tipo de formação vegetal (HOSOKAWA; SOLTER, 1995; OLIVEIRA et al., 2005; SOUZA et al., 2006). De acordo com Costa et al. (2002) e Coraiola; Péllico Netto (2003), tal conhecimento constitui o aspecto mais importante para a implantação de qualquer plano de manejo desses recursos.

O objetivo deste estudo foi comparar as mudanças ocorridas, devido à exploração florestal, na riqueza florística e diversidade de espécies em uma área ecotonal na região norte do estado de Mato Grosso, comparando a área que sofreu exploração florestal com a que não sofreu, testando a hipótese de que a exploração acarreta mudanças na riqueza florística e na diversidade de espécies da floresta.

\section{MATERIAIS E MÉTODOS}

\section{Caracterização da área}

A área de estudo localiza-se na Estação Experimental Pedro Nonato da Conceição, da Universidade Federal de Mato Grosso em cooperação com a Tecanorte Empreendimentos Florestais Ltda., nas coordenadas de $10^{\circ} 36^{\prime} 03^{\prime \prime}$ e $11^{\circ} 07^{\prime} 35^{\prime \prime}$ de latitude sul e $53^{\circ} 25^{\prime} 50^{\prime \prime}$ e $54^{\circ} 03^{\prime} 40^{\prime \prime}$ de longitude oeste, no município de Marcelândia, distante aproximadamente $900 \mathrm{~km}$ de Cuiabá, MT. Essa área é integrante do Projeto de Apoio ao Manejo Florestal Sustentável na Amazônia (ProManejo) MMA/IBAMA/PPG7 - Projeto Huaia-Missu.

O clima da região é do tipo AM de Köppen (clima tropical monçônico). A temperatura média anual é de $28^{\circ} \mathrm{C}$. A estação chuvosa é bem definida, entre os meses de outubro e março, e a estação seca entre abril e setembro. As precipitações anuais são entre 2.250 e $2.550 \mathrm{~mm}$.

Os solos são classificados como Latossolos Vermelho/Amarelo distróficos, de textura média, que predominam associados a Latossolos Vermelho Escuro distróficos, de textura média, e Areias Quartzosas distróficas (BRASIL, 1981).

Segundo Brasil (1981), trata-se de uma área de tensão ecológica, na região de contato entre Floresta Ombrófila e Floresta Estacional, com predominância da formação denominada Floresta Semidecidual, Submontana, dossel emergente. Nessas áreas, no período de seca, embora a maioria das espécies componentes do dossel arbóreo seja de árvores tipicamente amazônicas, cerca de $20 \%$ dos indivíduos perdem pelo menos parcialmente suas folhas, o que possibilita classificá-la como Floresta Estacional.

\section{Fonte de dados}

Os dados utilizados são provenientes de 74 parcelas permanentes com dimensões de $10 \mathrm{~m}$ x $250 \mathrm{~m}$, totalizando uma amostra com 18,5 ha. O método de amostragem utilizado foi o de área fixa, e o processo de amostragem empregado para instalação das parcelas foi o sistemático em múltiplos estágios, em que a primeira amostra foi selecionada aleatoriamente e as demais de forma sistemática, com distâncias fixas entre amostras na linha e entre linhas, conforme descrito por Péllico Netto; Brena (1997).

O levantamento amostral se deu em três ocasiões: a primeira medição ocorreu antes da exploração no segundo semestre de 2001, a segunda logo após a exploração, em 2003, e a terceira ocorreu quatro anos após a exploração, em 2007.

Das 74 parcelas amostrais instaladas em 2001, cinco sofreram exploração florestal em 2002, com a retirada das árvores de espécies e diâmetros comercialmente aceitos no mercado. A exploração ocorreu em uma unidade de produção com 240 ha e na intensidade máxima de $30 \mathrm{~m}^{3} / \mathrm{ha}$. Na área explorada havia cinco parcelas amostrais permanentes com a finalidade de refletir, pelas medições, as mudanças que ocorrem na floresta. As alterações nas áreas ocorrem em função de dois fatores: pela intervenção/exploração e pela própria dinâmica da floresta. Com este estudo, visou-se avaliar o grau de 
comprometimento da exploração e, por essa razão, fez-se, também, necessário comparar com área não explorada.

Em cada parcela amostral, todas as árvores com dap (diâmetro medido a 1,3 m de altura do solo) maior ou igual a $17 \mathrm{~cm}$ foram medidas, identificadas e plaqueteadas. As que apresentaram irregularidades, como sapopemas, nós, calosidades, podridão, danos ou qualquer deformação a 1,3 m, tiveram o ponto de medição feito em outra posição no fuste, a fim de evitar influência dessas irregularidades. Esses pontos floram marcados para posterior remedição.

Ressalte-se que o diâmetro de inclusão ( $d a p \geq 17 \mathrm{~cm}$ ), embora não seja usual, foi adotado em função do propósito do Projeto Huaia-Missu, que tem como fundamento o manejo de plantas estabelecidas e com potencialidade de uso comercial.

As remedições das parcelas foram efetuadas sempre no período de seca, e a remarcação do ponto de medição, reemplaqueteamento e checagem dos nomes científicos das espécies foram realizados nessas ocasiões.

Árvores mortas (mortalidade), bem como os indivíduos que passaram a atingir o tamanho mínimo de medição a cada ocasião (ingresso), foram registradas e computadas. O reconhecimento da identidade botânica dos indivíduos foi realizado, na floresta, por mateiros experientes da região. Paralelamente ao reconhecimento, o material botânico de algumas espécies não identificadas em campo foi coletado para posterior identificação, com o apoio do Herbário Central da Universidade Federal de Mato Grosso, onde as exsicatas foram depositadas.

\section{Processamento e análise dos dados}

Elaborou-se uma lista de todas as espécies arbóreas ocorrentes na área de estudo, contendo nome comum, nome científico, família e uso madeireiro, assim como o número de gêneros e espécies. A lista foi elaborada de forma geral para as duas áreas, a explorada e a não explorada, uma vez que as mesmas espécies ocorrem em ambas as áreas. A atribuição do uso madeireiro a cada espécie da área foi baseado nas espécies comercializadas da região.

A identificação taxonômica das espécies avaliadas foi efetuada mediante consultas a herbários, consultas a especialistas e por meio de literatura especializada. A sinonímia e a grafia dos taxa foram atualizadas mediante consulta ao índice de espécies do Royal Botanic Garden e ao banco de dados do Missouri Botanical Garden, disponível na página <http://mobot.mobot.org/W3T/Search/vast.html>. O sistema de classificação adotado foi o APG II (2003), exceto para Fabaceae, a qual foi considerada com as três subfamílias (Caesalpinioideae, Faboideae e Mimosoideae).

A diversidade florística da área, em cada medição, foi avaliada por meio do quociente de mistura de Jentsch e pelo índice de Shannon-Wiener (MAGURRAN, 1988) e a equabilidade pelo índice de Pielou (PIELOU, 1975):

$$
\mathrm{QM}=\frac{\mathrm{S}}{\mathrm{N}} \quad \mathrm{H}^{\prime}=-\sum_{\mathrm{i}=1}^{\mathrm{n}} \mathrm{p}_{\mathrm{i}} \times \ln \left(\mathrm{p}_{\mathrm{i}}\right) \quad \mathrm{J}^{\prime}=\frac{\mathrm{H}^{\prime}}{\mathrm{H}_{\text {máx }}^{\prime}}
$$

Em que: $\mathrm{QM}=$ quociente de mistura de Jentsch;

H'= índice de diversidade de Shannon;

J' = índice de Pielou;

$\mathrm{S}=\mathrm{n}^{\mathrm{o}}$ total de espécies amostradas na área;

$\mathrm{N}=\mathrm{n}^{\mathrm{o}}$ total de indivíduos amostrados na área;

$\mathrm{p}_{\mathrm{i}}=(\mathrm{ni} \div \mathrm{N})$, probabilidade de que um indivíduo amostrado pertença à espécie $\mathrm{i}$;

$\mathrm{n}_{\mathrm{i}}=\mathrm{n}^{\mathrm{o}}$ total de indivíduos da espécie $\mathrm{i}$;

$\mathrm{H}_{\text {máx }}=$ índice de diversidade de Shannon máximo $\left[\mathrm{H}^{\prime}{ }_{\max }=\ln (\mathrm{S})\right]$.

\section{RESULTADOS E DISCUSSÃO}

Na tabela 1 estão listadas todas as espécies, bem como as respectivas famílias, nomes científicos e populares e uso madeireiro na região, nos três levantamentos realizados na área não explorada e explorada, resultando em indidíduos distribuídos em 35 famílias botânicas, 77 gêneros e 98 espécies, que ocorreram na área de estudo, incluindo área explorada e não explorada. 
Tabela 1. Caracterização das espécies por famílias, nomes científicos e populares, uso, levantamento amostral e área de ocorrência.

Table 1. Characterization of the species by families, scientific and popular names, use, survey sample, and area of occurrence.

\begin{tabular}{|c|c|c|c|c|c|}
\hline Família & Nome científico & Nome popular & Uso* & Levantamento* & Área* \\
\hline \multirow[t]{2}{*}{ Anacardiaceae } & Astronium fraxinifolium Schott ex Spreng. & Muiracatiara & 1 & I, II e III & $\mathrm{NE}, \mathrm{E}$ \\
\hline & Spondias dulcis Parkinson & Cajarana & 3 & I, II e III & $\mathrm{NE}$ \\
\hline \multirow[t]{7}{*}{ Annonaceae } & Anonna mисоsa Jacq. & Pinha-da-mata & 3 & I, II e III & $\mathrm{NE}$ \\
\hline & Guatteria australis A. St.-Hil. & Pindaíba-preta & 3 & I, II e III & $\mathrm{NE}$ \\
\hline & Xylopia aromatica (Lam.) Mart. & Pimenta-de-macaco & 3 & I, II e III & $\mathrm{NE}$ \\
\hline & Xylopia benthamii R.E.Fr & Embireira & 3 & I, II e III & $\mathrm{NE}$ \\
\hline & Xylopia frutescens Aubl. & Pindaíba-branca & 3 & I, II e III & NE, E \\
\hline & Xylopia laevigata R.E.Fr. & Pindaíba-vermelha & 3 & I, II e III & $\mathrm{NE}$ \\
\hline & Xylopia sp. & Pindaíba & 3 & I, II e III & $\mathrm{NE}, \mathrm{E}$ \\
\hline \multirow[t]{6}{*}{ Apocynaceae } & Aspidosperma discolor A. DC. & Guarantã & 3 & I, II e III & $\mathrm{NE}, \mathrm{E}$ \\
\hline & Aspidosperma macrocarpon Mart. & Peroba-mica & 1 & I, II e III & $\mathrm{NE}$ \\
\hline & Aspidosperma parvifolium A. DC. & Guatambu & 3 & I e III & $\mathrm{NE}$ \\
\hline & Aspidosperma sp. & Peroba & 1 & I, II e III & $\mathrm{NE}, \mathrm{E}$ \\
\hline & Couma utilis (Mart.) Müll.Arg & Sorveira & 1 & I, II e III & $\mathrm{NE}$ \\
\hline & $\begin{array}{l}\text { Himatanthus sucuиba (Spruce ex Müll. Arg.) } \\
\text { Woodson }\end{array}$ & Sucuúba & 3 & I, II e III & $\mathrm{NE}$ \\
\hline Araliaceae & $\begin{array}{l}\text { Schefflera morototoni (Aubl.) Maguire, } \\
\text { Steyerm.\&Frodin }\end{array}$ & Mandiocão & 2 & I, II e III & $\mathrm{NE}$ \\
\hline \multirow[t]{2}{*}{ Bignoniaceae } & Jacaranda copaia (Aubl.) D. Don & Caroba & 2 & I, II e III & $\mathrm{NE}, \mathrm{E}$ \\
\hline & Tabebuia sp. & Ipê & 1 & I, II e III & $\mathrm{NE}$ \\
\hline \multirow[t]{4}{*}{ Burseraceae } & Protium robustum (Swart) D.M. Porter & Amescla-aroeira & 1 & I, II e III & $\mathrm{NE}$ \\
\hline & Protium sp. & Amescla-breu & 1 & I, II e III & $\mathrm{NE}, \mathrm{E}$ \\
\hline & Trattinnickia burserifolia Mart. & Morcegueira & 2 & I, II e III & $\mathrm{NE}, \mathrm{E}$ \\
\hline & Trattinnickia rhoifolia var. sprucei Engl. & Amescla & 2 & I, II e III & $\mathrm{NE}, \mathrm{E}$ \\
\hline \multirow[t]{2}{*}{ Clusiaceae } & Calophyllum brasiliense Cambess. & Guanandi & 2 & I, II e III & $\mathrm{NE}$ \\
\hline & Vismia cayennensis (Jacq.) Pers. & Lacre-da-mata & 3 & I, II e III & NE \\
\hline Cordiaceae & Cordia sp. & Louro-seda & 3 & I, II e III & $\mathrm{NE}, \mathrm{E}$ \\
\hline Combretaceae & Buchenavia sp. & Mirindiba & 1 & I, II e III & $\mathrm{NE}, \mathrm{E}$ \\
\hline \multirow[t]{2}{*}{ Elaeocarpaceae } & Sloanea grandiflora Earle Sm. & Pateiro & 3 & I, II e III & NEE \\
\hline & Sloanea sp. & Urucurana & 3 & I, II e III & $\mathrm{NE}$ \\
\hline \multirow[t]{5}{*}{ Euphorbiaceae } & Alchornea glandulosa Poepp. \& Endl. & Iricurana & 3 & I e II & $\mathrm{NE}$ \\
\hline & $\begin{array}{l}\text { Hevea brasiliensis (Willd. ex A. Juss.) Müll. } \\
\text { Arg. }\end{array}$ & Seringueira & 3 & I, II e III & $\mathrm{NE}$ \\
\hline & Mabea sp. & Mamoninha-da-mata & 3 & I, II e III & $\mathrm{NE}, \mathrm{E}$ \\
\hline & Sapium glandulosum (L.) Morong & Leiteiro & 3 & I, II e III & $\mathrm{NE}, \mathrm{E}$ \\
\hline & Sapium marmieri Huber & Burra-leiteira & 1 & I, II e III & $\mathrm{NE}$ \\
\hline \multirow[t]{6}{*}{ Fabaceae - Caes } & Apuleia leiocarpa (Vogel) J.F. Macbr & Garapeira & 1 & I, II e III & $\mathrm{NE}$ \\
\hline & Copaifera sp. & Copaíba & 1 & I, II e III & $\mathrm{NE}$ \\
\hline & Dialium guianense (Aubl.) Sandwith & Jutaí-pororoca & 3 & I, II e III & $\mathrm{NE}$ \\
\hline & Hymenaea courbaril L. & Jatobá & 1 & I, II e III & $\mathrm{NE}$ \\
\hline & Peltogyne sp. & Roxinho & 1 & I, II e III & $\mathrm{NE}, \mathrm{E}$ \\
\hline & Sclerolobium paniculatum Voegel & Tachi & 1 & I, II e III & $\mathrm{NE}, \mathrm{E}$ \\
\hline \multirow[t]{8}{*}{ Fabaceae - Fabo } & Alexa grandiflora Ducke & Melancieira & 2 & I e II & NE \\
\hline & Andira surinamensis (Bondt) Splitg. ex Pulle & Angelim-manteiga & 1 & I, II e III & $\mathrm{NE}$ \\
\hline & Bowdichia nitida Spruce ex Benth. & Sucupira-amarela & 1 & I, II e III & $\mathrm{E}$ \\
\hline & Bowdichia sp. & Sucupira & 1 & I, II e III & NE \\
\hline & Bowdichia virgilioides Kunth & Sucupira-preta & 1 & I, II e III & $\mathrm{NE}, \mathrm{E}$ \\
\hline & Dipteryx odorata (Aubl.) Willd. & Cumaru & 1 & I, II e III & NE, E \\
\hline & Hymenolobium sp. & Angelim & 1 & I, II e III & $\mathrm{NE}$ \\
\hline & Myroxylon peruiferum L.f. & Pau-sangue & 3 & I, II e III & NE, E \\
\hline
\end{tabular}




\begin{tabular}{|c|c|c|c|c|c|}
\hline & Pterocarpus sp. & Pau-veneno & 3 & I, II e III & NE, E \\
\hline & Vatairea sericea (Ducke) Ducke & Angelim-amargoso & 1 & I, II e III & NE \\
\hline \multirow[t]{8}{*}{ Fabaceae - Mimo } & Abarema jupunba (Willd.) Britton\&Killip & Saboeiro & 2 & I, II e III & $\mathrm{NE}$ \\
\hline & Albizia hassleri (Chodat) Burkart & Farinha-seca & 2 & I, II e III & NE, E \\
\hline & Dinizia excelsa Ducke & Angelim-pedra & 1 & I, II e III & NE, E \\
\hline & Enterolobium maximum Ducke & Tamboril & 1 & I, II e III & NE, E \\
\hline & Inga paraensis Ducke & Ingá-vermelho & 3 & I, II e III & $\mathrm{NE}$ \\
\hline & Inga sp. & Ingá & 3 & I, II e III & $\mathrm{NE}, \mathrm{E}$ \\
\hline & Parkia pendula (Willd.) Benth. Ex Walp. & Angelim-saia & 2 & I, II e III & NE, E \\
\hline & $\begin{array}{l}\text { Zygia racemosa (Ducke) Barneby \& J.W. } \\
\text { Grimes }\end{array}$ & Angelim-rajado & 1 & I, II e III & NE \\
\hline Goupiaceae & Goupia glabra Aubl. & Cupiúba & 1 & I, II e III & $\mathrm{NE}$ \\
\hline Lamiaceae & Vitex sp. & Tarumarana & 3 & I & $\mathrm{NE}$ \\
\hline \multirow[t]{5}{*}{ Lauraceae } & Mezilaurus itauba (Meisn.) Taub. ex Mez & Itaúba & 1 & I, II e III & $\mathrm{NE}, \mathrm{E}$ \\
\hline & Nectandra cuspidata Nees \& Mart. & Canelão & 1 & I, II e III & $\mathrm{NE}, \mathrm{E}$ \\
\hline & Ocotea aciphylla (Nees) Mez & Canela-branca & 1 & I, II e III & $\mathrm{NE}$ \\
\hline & Ocotea pauciflora (Nees) Mez & Canelinha & 3 & I & $\mathrm{NE}$ \\
\hline & Ocotea sp. & Canela & 1 & I, II e III & NE, E \\
\hline \multirow[t]{3}{*}{ Lecythidaceae } & Cariniana sp. & Jequitibá & 1 & I e II & $\mathrm{NE}$ \\
\hline & Couratari sp. & Tauari & 1 & I, II e III & $\mathrm{NE}$ \\
\hline & Eschweilera sp. & Matamatá & 3 & I, II e III & $\mathrm{NE}, \mathrm{E}$ \\
\hline \multirow[t]{2}{*}{ Lythraceae } & Lafoensia pacari A.St.-Hil. & Amarelinho & 3 & I, II e III & $\mathrm{NE}$ \\
\hline & Physocalymma scaberrimum Pohl & Aricá & 3 & I, II e III & NE \\
\hline Malpighiaceae & Byrsonima densa (Poir) DC. & Murici & 1 & I, II e III & $\mathrm{NE}$ \\
\hline \multirow[t]{3}{*}{ Malvaceae } & Apeiba tibourbou Aubl. & Escova-de-macaco & 3 & I, II e III & $\mathrm{NE}, \mathrm{E}$ \\
\hline & Ceiba pentandra (L.) Gaertn. & Sumaúma & 2 & I, II e III & $\mathrm{NE}, \mathrm{E}$ \\
\hline & Sterculia apetala (Jacq.) H .Karst. & Manduvi & 3 & I, II e III & $\mathrm{E}$ \\
\hline \multirow[t]{2}{*}{ Melastomataceae } & Bellucia grossularioides (L.) Triana & Goiaba-de-anta & 3 & I, II e III & $\mathrm{NE}$ \\
\hline & Miconia ferruginata DC. & Miconia & 3 & I, II e III & NE, E \\
\hline Meliaceae & Guarea silvatica C. DC. & Cedro-marinheiro & 1 & I, II e III & $\mathrm{NE}$ \\
\hline Memecylaceae & Mouriri sp. & Canela-de-cutia & 3 & I, II e III & $\mathrm{NE}, \mathrm{E}$ \\
\hline \multirow[t]{4}{*}{ Moraceae } & Ficus sp. & Figueira & 2 & I, II e III & $\mathrm{NE}, \mathrm{E}$ \\
\hline & Helicostylis pedunculata Benoist & Inharé & 2 & I, II e III & $\mathrm{NE}$ \\
\hline & Maclura tinctoria (L.) D. Don ex Steud. & Amoreira & 3 & I, II e III & NE \\
\hline & $\begin{array}{l}\text { Maquira calophylla (Poepp. \& Endl.) C.C. } \\
\text { Berg }\end{array}$ & Cega-corrente & 3 & I, II e III & NE, E \\
\hline \multirow[t]{2}{*}{ Myrtaceae } & Eugenia lambertiana DC. & Goiabinha & 3 & I, II e III & NE, E \\
\hline & Eugenia paraensis O. Berg & Araçá & 3 & I, II e III & $\mathrm{NE}$ \\
\hline Ochnaceae & Ouratea sp. & Ouratea & 3 & I, II e III & $\mathrm{NE}, \mathrm{E}$ \\
\hline Rubiaceae & Amaioua guianensis Aubl. & Marmelada & 3 & I, II e III & $\mathrm{NE}, \mathrm{E}$ \\
\hline Rutaceae & Zanthoxylum rhoifolium Lam. & Mamica-de-porca & 3 & I, II e III & $\mathrm{NE}$ \\
\hline Salicaceae & Casearia sp. & Espeteiro & 3 & I, II e III & $\mathrm{NE}$ \\
\hline Sapindaceae & Matayba arborescens (Aubl.) Radlk. & Breu-de-tucano & 3 & I, II e III & $\mathrm{NE}, \mathrm{E}$ \\
\hline \multirow[t]{5}{*}{ Sapotaceae } & Chrysophyllum lucentifolium Cronq. & Goiabão & 3 & I, II e III & $\mathrm{E}$ \\
\hline & Manilkara excelsa (Ducke) Standl. & Maçaranduba & 1 & I, II e III & NE \\
\hline & Manilkara sp. & Balata & 3 & I, II e III & NE, E \\
\hline & Micropholis melinoniana Pierre & Pau-sapo & 3 & I, II e III & NE, E \\
\hline & Pouteria macrophylla (Lam.) Eyma & Tuturubá & 3 & I, II e III & $\mathrm{NE}$ \\
\hline Simaroubaceae & Simarouba amara Aubl. & Caixeta & 1 & I, II e III & $\mathrm{NE}$ \\
\hline Urticaceae & Cecropia sp. & Embaúba & 3 & I, II e III & NE, E \\
\hline \multirow[t]{3}{*}{ Vochysiaceae } & Vochysia divergens Pohl & Cambará & 1 & I, II e III & NE, E \\
\hline & Vochysia haenkeana Mart. & Cambará-liso & 1 & I, II e III & $\mathrm{NE}$ \\
\hline & Vochysia sp. & Cambará-rosa & 1 & I, II e III & $\mathrm{NE}, \mathrm{E}$ \\
\hline
\end{tabular}

* 1: serraria; 2: laminação; 3: sem uso madeireiro definido; I: inventário realizado em 2001; II: inventário realizado em 2003; III: inventário realizado em 2007; NE: área não explorada; E: área explorada.

FLORESTA, Curitiba, PR, v. 41, n. 2, p. 295-304, abr./jun. 2011. 
Ferreira Júnior et al. (2008), avaliando a composição, diversidade e similaridade florística desta área em estudo, amostrando todas as plantas lenhosas eretas com circunferência do caule a 1,3 m de altura do solo (CAP) maior ou igual a $45 \mathrm{~cm}$, registraram uma densidade de aproximadamente 216 indivíduos/ha, totalizando 4008 indivíduos arbóreos distribuídos em 33 famílias e 92 espécies.

$\mathrm{Na}$ área não explorada, foram registrados 4071,4175 e 4315 indivíduos com DAP $\geq 17 \mathrm{~cm}$ nos levantamentos efetuados em 2001, 2003 e 2007, respectivamente.

Nas parcelas exploradas em 2001, foram registrados 329 indivíduos com DAP $\geq 17 \mathrm{~cm}$. Em 2003, dois anos após a exploração, e em 2007, quatro anos após a exploração, foram registrados, respectivamente, 332 e 430 indivíduos com DAP $\geq 17 \mathrm{~cm}$ nas cinco parcelas amostradas. O número de famílias, gêneros e espécies permaneceu o mesmo que em 2001.

Analisando a riqueza florística nas duas áreas, verificou-se que exploração florestal não demonstrou evidências de ocasionar mudanças na mesma.

Francez et al. (2007), avaliando as mudanças ocorridas na composição florística em uma área de 9 ha de floresta de terra firme na região de Paragominas, PA, registraram 4469 indivíduos com $\mathrm{DAP} \geq 10 \mathrm{~cm}$, distribuídos em 46 famílias, 138 gêneros e 228 espécies na área não explorada e 4330 indivíduos em 46 famílias, 138 gêneros e 226 espécies um ano após a exploração florestal, verificando que a composição florística sofreu alterações significativas devido à exploração de impacto reduzido a que foi submetida.

Lima et al. (2007), analisando a estrutura de uma área de $4000 \mathrm{~m}^{2}$ de floresta secundária na região de Manaus, $\mathrm{AM}$, adotanto como critério de inclusão indivíduos com DAP $\geq 5 \mathrm{~cm}$, registraram, 10 anos após corte raso seguido de fogo, 532 indivíduos, 41 famílias e 138 espécies antes da exploração e 459 indivíduos, 34 famílias e 53 espécies após a exploração. Esses autores verificaram que, dez anos após a queimada, a floresta secundária ainda era bastante diferente da floresta original.

As famílias mais ricas em espécies (Tabela 2) foram: Lauraceae, Sapotaceae, Burseraceae, Melastomataceae e Fabaceae/Mimosoideae (representando mais de 50\% do total de espécies) na área não explorada e Sapotaceae, Lauraceae, Burseraceae, Fabaceae/Mimosoideae, Melastomataceae e Vochysiaceae (representando mais de 50\% do total de espécies) na área explorada, indicando predomínio dessas famílias na área.

Tabela 2. Abundância das principais famílias ocorrentes na área não explorada e explorada nos levantamentos realizados em 2001, 2003 e 2007.

Table 2. Abundance of the main families occurring in the area not explored and explored in surveys performed in 2001, 2003, and 2007.

\begin{tabular}{|c|c|c|c|c|c|c|c|}
\hline \multicolumn{4}{|c|}{ Área não explorada } & \multicolumn{4}{|c|}{ Área explorada } \\
\hline Famílias & 2001 & 2003 & 2007 & Famílias & 2001 & 2003 & 2007 \\
\hline Lauraceae & $15,6 \%$ & $15,5 \%$ & $15,6 \%$ & Sapotaceae & $13,1 \%$ & $13,0 \%$ & $12,9 \%$ \\
\hline Sapotaceae & $11,4 \%$ & $11,4 \%$ & $11,3 \%$ & Lauraceae & $10,6 \%$ & $10,5 \%$ & $10,6 \%$ \\
\hline Burseraceae & $8,7 \%$ & $8,7 \%$ & $8,8 \%$ & Burseraceae & $9,7 \%$ & $9,9 \%$ & $10,0 \%$ \\
\hline Melastomataceae & $8,3 \%$ & $8,3 \%$ & $8,3 \%$ & Fabaceae/Mimosoideae & $9,7 \%$ & $9,9 \%$ & $10,0 \%$ \\
\hline Fabaceae/Mimosoideae & $8,2 \%$ & $8,2 \%$ & $8,0 \%$ & Melastomataceae & $5,5 \%$ & $5,4 \%$ & $5,5 \%$ \\
\hline Fabaceae/Caesalpinioideae & $6,4 \%$ & $6,3 \%$ & $6,5 \%$ & Vochysiaceae & $4,9 \%$ & $4,8 \%$ & $4,7 \%$ \\
\hline Euphorbiaceae & $4,3 \%$ & $4,4 \%$ & $4,3 \%$ & Annonaceae & $4,6 \%$ & $4,8 \%$ & $5,0 \%$ \\
\hline Apocynaceae & $4,0 \%$ & $4,0 \%$ & $3,9 \%$ & Apocynaceae & $4,6 \%$ & $4,5 \%$ & $4,4 \%$ \\
\hline Vochysiaceae & $4,0 \%$ & $4,0 \%$ & $4,0 \%$ & Moraceae & $4,3 \%$ & $4,2 \%$ & $4,4 \%$ \\
\hline Annonaceae & $3,4 \%$ & $3,4 \%$ & $3,5 \%$ & Fabaceae/Faboideae & $3,7 \%$ & $3,6 \%$ & $3,5 \%$ \\
\hline Demais famílias & $25,9 \%$ & $26,1 \%$ & $25,9 \%$ & Demais famílias & $29,4 \%$ & $29,2 \%$ & $28,9 \%$ \\
\hline
\end{tabular}

Os gêneros mais importantes foram Ocotea e Manilkara, respectivamente, nas áreas não explorada e explorada (Tabela 3).

Francez et al. (2007) destacaram as famílias Fabaceae, Lecythidaceae, Sapotaceae e Violaceae que, juntas, constituíram 66,3\% de abundância da área não explorada. No presente estudo, as famílias Sapotaceae, Mimosaceae, Annonaceae, Caesalpiaceae e Lauraceae também se destacaram quanto ao número de indivíduos, entretanto Chrysobalanaceae e Lecythidaceae não ocorreram entre as dez mais abundantes, e a família Violaceae não ocorreu na área. 
Os valores do coeficiente de mistura, do índice de Shannon e de equabilidade são apresentados na tabela 4 .

Tabela 3. Gêneros mais importantes ocorrentes na área não explorada e explorada nos levantamentos realizados em 2001, 2003 e 2007.

Table 3. Most important generas occurring in the area not explored and explored in surveys performed in 2001, 2003 and 2007.

\begin{tabular}{lccclccc}
\hline \multicolumn{4}{c}{ Área não explorada } & \multicolumn{4}{c}{ Área explorada } \\
\hline Gêneros & $\mathbf{2 0 0 1}$ & $\mathbf{2 0 0 3}$ & $\mathbf{2 0 0 7}$ & Gêneros & $\mathbf{2 0 0 1}$ & $\mathbf{2 0 0 3}$ & $\mathbf{2 0 0 7}$ \\
\hline Ocotea & $8,4 \%$ & $8,3 \%$ & $8,2 \%$ & Manilkara & $9,3 \%$ & $10,3 \%$ & $10,2 \%$ \\
Manilkara & $7,8 \%$ & $7,9 \%$ & $8,0 \%$ & Trattinnickia & $7,5 \%$ & $5,3 \%$ & $6,1 \%$ \\
Trattinnickia & $5,7 \%$ & $5,7 \%$ & $5,9 \%$ & Aspidosperma & $6,2 \%$ & $7,4 \%$ & $7,9 \%$ \\
Aspidosperma & $5,6 \%$ & $5,7 \%$ & $5,8 \%$ & Ocotea & $5,6 \%$ & $6,1 \%$ & $5,8 \%$ \\
Miconia & $4,7 \%$ & $4,8 \%$ & $4,7 \%$ & Vochysia & $5,2 \%$ & $5,5 \%$ & $5,3 \%$ \\
Mouriri & $4,4 \%$ & $4,3 \%$ & $4,4 \%$ & Inga & $4,3 \%$ & $4,0 \%$ & $3,8 \%$ \\
Peltogyne & $4,2 \%$ & $4,2 \%$ & $4,4 \%$ & Xylopia & $3,9 \%$ & $3,8 \%$ & - \\
Albizia & $3,5 \%$ & $3,6 \%$ & $3,5 \%$ & Miconia & $3,8 \%$ & $4,2 \%$ & $4,0 \%$ \\
Mezilaurus & $3,5 \%$ & $3,6 \%$ & $3,6 \%$ & Apeiba & $3,8 \%$ & $4,3 \%$ & $4,5 \%$ \\
Sapium & $3,2 \%$ & $3,2 \%$ & $3,1 \%$ & Albizia & $3,5 \%$ & $3,8 \%$ & $4,1 \%$ \\
& & & & Maquira & - & - & $3,7 \%$ \\
\hline
\end{tabular}

Tabela 4. Quociente de mistura de Jentsch, índices de diversidade de Shanon e equabilidade de Pielou de espécies arbóreas ocorrentes na área não explorada e explorada nos levantamentos realizados em 2001, 2003 e 2007.

Table 4. Moisture exchange coefficient of Jentsch, the Shannon-Wiener diversity index, and equability by Pielou's index for three arboreal species occurring in the area not explored and explored in surveys performed in 2001, 2003, and 2007.

\begin{tabular}{lllllll}
\hline \multirow{2}{*}{ Índices } & \multicolumn{3}{c}{ Área não explorada } & \multicolumn{3}{c}{ Área explorada } \\
\cline { 2 - 7 } & $\mathbf{2 0 0 1}$ & $\mathbf{2 0 0 3}$ & $\mathbf{2 0 0 7}$ & $\mathbf{2 0 0 1}$ & $\mathbf{2 0 0 3}$ & $\mathbf{2 0 0 7}$ \\
\hline Quociente de mistura de Jentsch (QM) & $1: 3,8$ & $1: 3,6$ & $1: 3,7$ & $1: 8,0$ & $1: 8,0$ & $1: 8,2$ \\
Diversidade de Shannon (H') & 4,10 & 4,53 & 4,48 & 4,61 & 4,62 & 4,61 \\
Equabilidade (J') & 0,70 & 0,74 & 0,73 & 0,79 & 0,79 & 0,79 \\
\hline
\end{tabular}

O QM foi superior a 1:3 e a 1:8, respectivamente, para as áreas não explorada e explorada. De acordo com Finol (1975), o QM representaria alta heterogeneidade em florestas naturais tropicais quando fosse de de aproximadamente 9 indivíduos por espécie. Portanto, de acordo com os valores encontrados, pode-se afirmar que ocorre baixa heterogeneidade na área não explorada e média heterogeneidade na área explorada.

Os quocientes de Jentsch estimados estão abaixo dos encontrados por Higuchi et al. (1998) em uma floresta densa de terra firme pertencente à bacia rio Cueiras em dois transectos de cinco hectares cada, considerando DAP $\geq 10$, que foram de $1: 14$ e $1: 12$.

Francez et al. (2007) encontraram índice de Shannon de 4,27, valor esse inferior ao do presente estudo, porém a equabilidade encontrada em Paragominas foi maior, 0,81, indicando alta uniformidade nas proporções do número de indivíduos/número de espécies dentro da comunidade vegetal.

A composição florística no estudo de Higuchi et al. (1998) apresentou estimativas de H' de 4,39 e 4,59 nos transectos 1 e 2, e Francez et al. (2007) obtiveram 4,29 para Paragominas. Esses valores são semelhantes aos encontrados na área de estudo. Ribeiro et al. (1999) encontraram valores inferiores ao estudado, que foram de 3,66 e 3,71, para as regiões de Carajás e Marabá.

O índice de Shannon em florestas tropicais varia de 3,83 a 5,85 (KNIGHT, 1975), valores considerados altos para qualquer tipo de vegetação (OLIVEIRA; AMARAL, 2004). Verificou-se, portanto, que os valores de $H^{\prime}$ do presente estudo indicam tratar-se de áreas com diversidade relativamente alta.

O grau estimado de equitabilidade foram todos superiores a 0,7 , o que sugere alta uniformidade nas proporções do número de indivíduos/número de espécies dentro da comunidade vegetal, constatação

FLORESTA, Curitiba, PR, v. 41, n. 2, p. 295-304, abr./jun. 2011. 
esperada, pois a equitabilidade é diretamente proporcional à diversidade e antagônica à dominância (UHL; MURPHY, 1981). Teoricamente, esse valor indica que seria necessário o incremento de cerca de 30\% de espécies para atingir a diversidade máxima da comunidade vegetal, segundo Brower et al. (1998).

Os índices de equabilidade apresentaram valores superiores aos encontrados por Higuchi $e t$ al. (1998) nos dois transectos, 0,60 e 0,58, indicando que as espécies da área de estudo possuem distribuição mais unifome. No entanto, quando comparada ao estudo de Francez et al. (2007) - 0,79 de índice de equabilidade -, a distribuição uniforme se torna ligeiramente inferior.

\section{CONCLUSÕES}

Apesar de os levantamentos terem ocorrido pouco tempo (dois e cinco anos) após a exploração florestal, verificou-se que não há evidências de que a exploração tenha ocasionado mudanças na riqueza florística e na diversidade de espécies da floresta, rejeitando-se a hipótese testada.

\section{REFERÊNCIAS}

APG II. An update of the Angiosperm Phylogeny Group classification for the orders and families of flowering plants: APG II. Botanical Journal of the Linnean Society, v. 141, n. 4, p. 399 - 436, 2003.

BRASIL. Ministério das Minas e Energia. Departamento Nacional de Produção Mineral. Projeto RADAMBRASIL: Volume 22 Folha Sc 22 - Tocantins. Rio de Janeiro, 1981.

BROWER, J. E.; ZAR, J. H.; VAN ENDE, C. N. Field and laboratory methods for general ecology. 4. ed. New York: WCB/McGraw, 1998. 273 p.

CORAIOLA, M.; PÉLLICO NETTO, S. Levantamento da composição florística de uma floresta estacional semidecidual localizada no município de Cássia, MG. Acadêmica: Ciências Agrárias e Ambientais, v. 1, n. 1, p. 11 - 21, 2003.

COSTA, D. H. M.; CARVALHO, J. O. P.; SILVA, J. N. M. Dinâmica da composição florística após a colheita de madeira em uma área de terra firme na Floresta Nacional do Tapajós (PA). Ciências Agrárias, v. 38, p. 67 - 90, 2002.

FERREIRA JÚNIOR, E. V.; COSTA, M. F. F.; SOARES, T. S.; SILVA, V. S. M. Composição, diversidade e similaridade florística de uma floresta tropical semidecídua submontana em Marcelândia, MT. Acta Amazônica, v. 38, n. 4, p. 673 - 680, 2008.

FRANCEZ, L. M. B.; CARVALHO, J. O. P.; JARDIM, F. C. S. Mudanças ocorridas na composição florística em decorrência da exploração florestal em uma área de floresta de terra firme na região de Paragominas, PA. Acta Amazônica, v. 37, n. 2, p. 219 - 228, 2007.

HIGUCHI, N.; CAMPOS, M. A. A.; SAMPAIO, P. T. B.; SANTOS, J. Pesquisas florestais para a conservação da floresta e reabilitação de áreas degradadas da Amazônia. Manaus: INPA, 1998. 264 p.

HOSOKAWA, R. T.; SOLTER, F. Manejo florestal. Curitiba: UFPR, 1995. 43 p.

KNIGHT, D. H. A phytosociological analysis of species-rich tropical forest on Barro Colorado Island, Panama. Ecological Monographs, v. 45, p. 259 - 284, 1975.

LIMA, A. J. N.; TEIXEIRA, L. M.; CARNEIRO, V. M. C.; SANTOS, J.; HIGUCHI, N. Análise da estrutura e estoque de fitomassa de uma floresta secundária da região de Manaus AM, dez anos após o corte raso seguido de fogo. Acta Amazônica, v. 37, n. 2, p. 49 - 54, 1999.

MAGURRAN, A. E. Ecological diversity and its measurement. Princeton: Princeton University, 1988. $192 \mathrm{p}$.

OLIVEIRA, A. N.; AMARAL, I. L. Florística e fitossociologia de uma floresta de vertente na Amazônia Central, Amazonas, Brasil. Acta Amazônica, v. 34, n. 1, 2004. 
OLIVEIRA, L. C.; COUTO, H. T. Z.; SILVA, J. N. M.; CARVALHO, J. O. P. Efeito da exploração de madeira e tratamentos silviculturais na composição florística e diversidade de espécies em uma área de 136 ha na Floresta Nacional do Tapajós, Belterra, Pará. Scientia Forestalis, Piracicaba, n. 69, p. 62 - 76, 2005.

PÉLLICO NETO, S.; BRENA, D. A. Inventário florestal. Curitiba: [s.e.], 1997. 316 p.

PIELOU, E. C. Ecological diversity. New York: John Wiley \& Sons Inc., 1975. 176 p.

RIBEIRO, R. J.; HIGUCHI, N.; SANTOS, J.; AZEVEDO, C. P. Estudos fitossociológicos nas regiões de Carajás e Marabá, Pará, Brasil. Acta Amazônica, v. 29, n. 2, p. 207 - 222, 1999.

SANQUETTA, C. R.; CUNHA, U. S.; WATZLAWICK, L. F.; CAMPOS, M. L. B. Projeção da distribuição diamétrica de fragmentos de florestas semidecíduas com matriz de transição. Ciências Exatas e Naturais, v. 3, n. 1, p. 75 - 85, 2001.

SOUZA, D. R.; SOUZA, A. L.; LEITE, H. G.; YARED, J. A. G. Análise estrutural em floresta ombrófila densa de terra firme não explorada, Amazônia Oriental. Revista Árvore, Viçosa, v. 30, n. 1, p. 75 - 87 , 2006.

UHL, C.; MURPHY, P. G. Composition, structure, and regeneration of a tierra firme forest in the Amazon Basin of Venezuela. Tropical Ecology, v. 22, n. 2, p. 219 - 237, 1981. 
